\title{
Piezo-Electric Activity of Rochelle Salt Under Various Conditions
}

BY

J. VALASEK

A THESIS

Submitted to the Faculty of the Graduate School of the University of MinNesota in PARTIAL FULFILLMENT OF THE REQUIREMENTS FOR THE DEgReE OF DOCTOR OF PHILOSOPHy. 


\title{
PIEZO-ELECTRIC ACTIVITY OF ROCHELLE SALT UNDER VARIOUS CONDITIONS.
}

\author{
By J. VAlasek.
}

\section{SyNopsis.}

Electrical Properties of Rockelle Sall Crystal are analogous to the magnetic properties of iron, the dielectric displacement $D$ and polarization $P$ varying with the electric field $E$ in the same general manner as $B$ and $I$ vary with $H$ for iron, and showing an electric hysteresis with loops distorted by an amount corresponding to the permanent polarization $P_{0}$, whose value is about $30 \mathrm{e} .8 . \mathrm{u} . / \mathrm{cm} .^{3}$ but varies for different crystals. The dielectric constant $(\kappa=d D / d E)$ was measured from $-70^{\circ}$ to $30^{\circ} \mathrm{C}$. and found to be surprisingly large, increasing from about 50 at $-70^{\circ}$ to a maximum of about 1,000 near $0^{\circ}$. The modulus of piezo-electric activity for shearing stresses $(\delta)$ varies with temperature, $-70^{\circ} 1040^{\circ} \mathrm{C}$., in a very similar manner, increasing from less than $10^{-6}$ at $-70^{\circ}$ to a maximum of about $10^{-6}$ at $0^{\circ}$. The ratio $\delta / \kappa$ varied with the electrode material, being greater for tin foil than for mercury electrodes. The difference may be due to the alcohol used in shellacking the tin-foil electrodes on. There are other indications that $\delta$ and $\kappa$ are related. The variation of $\delta$ with himidity is such as can be accounted for by the decrease in the dielectric constant of the outer layer as a result of dehydration. The change of polarization produced by pressure as measured by the change in the hysteresis loop agrees with the value found directly from the piezo-electric response, as required by Lord Kelvin's theory. Also fatigue effects on $\delta$ produced by temporarily applied fields are traceable to fatigue in the polarization. The electrical conductivity below $45^{\circ}$ is less than $5 \times \mathrm{ro}^{-7} \mathrm{mhos} / \mathrm{cm}^{3}$ but from $43^{\circ}$ to $57^{\circ}$ increases rapidly to $5 \times 10^{-4}$.

Optical Properties of Rochelle Salt as Calculated from the Natural Polarization.Assuming only one electron is displaced the natural period corresponds to a wavelength of $4.2 \mu$ and the specific rotation for sodium light comes out $10^{\circ}$, the observed value being $22^{\circ}$. 1 .

RECENTLY ${ }^{1}$ the writer described some experiments on the dielectric and piezo-electric properties of Rochelle salt, which were made for the purpose of correlating and explaining the effects observed chiefly by Cady and by Anderson. The plates used were cut with faces perpendicular to the $\mathrm{a}$ axis and with edges at $45^{\circ}$ with the $\bar{b}$ and $c$ axes. The present paper is a continuation of the work, the variations in the electrical properties having been studied more extensively. The apparatus and method of observation have been already described in the paper referred to above. The more important results obtained at that time can be summarized as follows:

In the case of Rochelle salt the dielectric displacement $D$, electric intensity $E$, and polarization $P$ behave in a manner analogous to $B$, $H$, and $I$ in the case of magnetism. Rochelle salt shows an electric

${ }^{1}$ J. Valasek, PHYs. REv. (2), XVII, p. 475. 
hysteresis in $P$ analogous to the magnetic hysteresis in the case of iron, the loops however being distorted by an amount corresponding to the permanent polanization of the crystal in the natural state. This point of view is very effective in accounting for many of the peculiarities observed.

In an electric field the piezo-electric activity has a maximum for a definite value of the field and decreases to a small value in both directions. The position of the maximum corresponds to the greatest rate of change of polarization with electric field in the case of the condenser experiments. In fact if force and electric field are equivalent in changing the piezoelectric polarization then the response for a given force in various applied fields must necessarily give curves of the same general nature as curves of $\partial P / \partial E$ or $\partial D / \partial E$ against $E$. It is permissible to interchange $D$ and $P$ in most cases because of the large dielectric constant of Rochelle salt.

\section{Relation between Polarization and Piezo-electric Activity.}

The activity of a piezo-electric crystal is intimately related to the natural polarization. According to Lord Kelvin this natural moment is masked by surface charges so that the crystal appears to be uncharged. This polarization or piezo-electric moment can be measured independently of the charges on the electrodes, through the distortion of the hysteresis loop. The center $A$ of the loop is found by a consideration of symmetry and may be assumed to represent the condition of no polarization. If the natural condition of polarization is assumed to be half way between the two branches of the loop at zero field then the value of the permanent polarization $P_{0}$ is proportional to $A B$, Fig. $\mathrm{x}$. There being

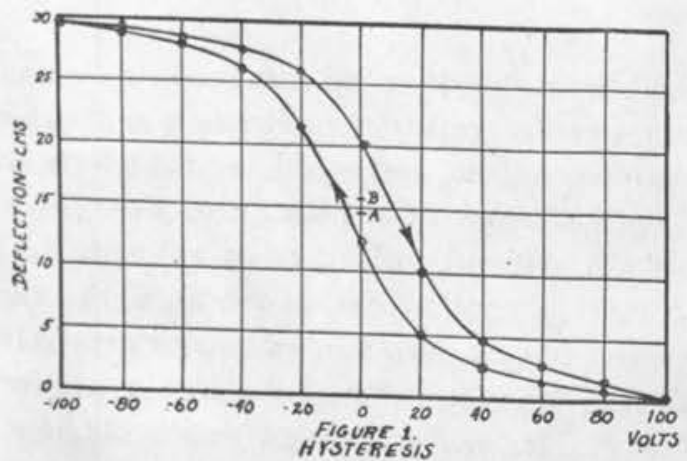

Fig. 1.

no field applied, the equation for the work done per unit charge carried through the condenser is:

$$
4 \pi\left(\frac{Q_{0}}{S}-P_{0}\right) t=0,
$$


sn that

$$
P_{0}=\frac{Q_{0}}{S}
$$

where $Q_{0}$ is the apparent average permanent charge at zero field given by $A B$ (Fig. I) and where $S$ is the area of the plate. Calculation gives the value: 30 e.s.v. $/ \mathrm{cm}^{2}$.

According to Lord Kelvin's theory an applied stress will change this polarization so as to create free charges on the electrodes. A force of 250 grams applied to the crystal should consequently shift the loop by an amount equivalent to the piezo-electric response for 250 grams. When this experiment was performed another, but more unsymmetrical loop, was obtained. The change in polarization by the loop method was II4 e.s.u./cm. ${ }^{2}$ while the piezo-electric response amounted to I2I e.s.u. $\mathrm{cm}^{2}$

The value of $P_{0}$ obtained from the hysteresis loops is only approximate because of the assumptions involved in its determination. It cannot, moreover, be fixed definitely enough to be put down as a physical constant of Rochelle salt because it varies with different specimens, besides changing with temperature, pressure and fatigue. The value $P_{0}=30$ e.s.u. $/ \mathrm{cm}^{2}{ }^{2}$ is thought to be a representative value and is checked by other measurements. The writer would not be surprised, however, to find other specimens giving several times this value. The change in polarization due to pressure however is derived by a differential method eliminating much of the uncertainty in measurements on one loop. The result in this case should be fairly definite, as indeed it seems to be.

Piezo-electric activity depends on both the crystalline structure and on the polarization. It is greatest for a polarization somewhat larger than normal and decreases in both directions for changes in this quantity, the polarization being changed by applying an electric field. It has been shown by the writer that this relation between activity and applied field is approximately like that of the derivative $\partial D / \partial E$ of the curve relating the dielectric displacement $D$ and the electric field $E$ of the crystal used as a condenser. Since this latter relation is in the form of a hysteresis loop it follows that the activity is also a double-valued function of the applied field depending on the direction of variation of the field. A curve illustrating this effect is reproduced in Fig. 2. The readings were taken in as short a time as possible to eliminate fatigue. These curves show that the piezo-electric response at zero field depends on the previous electrical treatment of the crystal. The latter fact has also been noted by W. G. Cady in the report previously referred to. 
This after-effect does not persist very long but dies off exponentially with the time. The piezo-electric response or ballistic throw of the galvanometer for 250 grams has been observed to return to half value in I minute and to normal in over 20 minutes after fields of 150 volts have been applied for 3 minutes previously. There is a much greater aftereffect in the direction of increased activity.

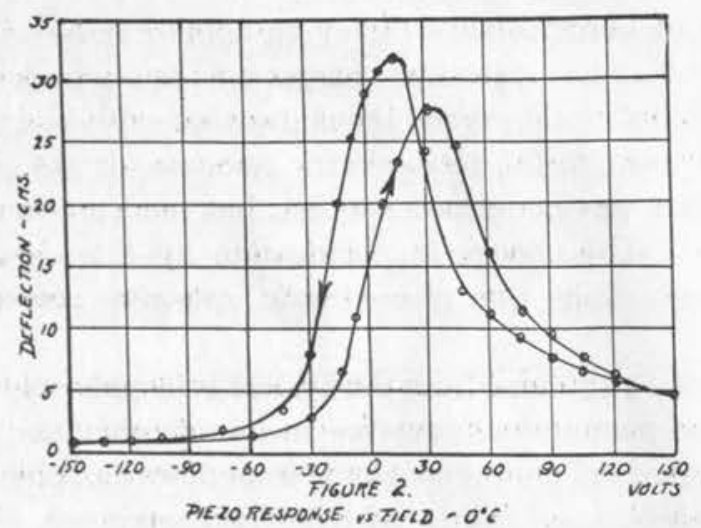

Fig. 2.

A corresponding dielectric effect is indicated by the double value of the condenser charge at zero field in the hysteresis loops. This is clearly due to a fatigue in the polarization and it also dies off exponentially with time. Herein is probably found the explanation of the "storage battery effect" described by W. G. Cady who observed that after applying a field of 100 volts for some time there was, on removal of the field, a small current which decreased gradually and flowed from the crystal as from a miniature storage battery.

The piezo-electric fatigue may well be a direct result of the fatigue in the polarization, as there seems to be a close relation between piezoelectric activity and polarization. It appears that the activity is approximately proportional to the rate of change of polarization with applied field and hence proportional to the dielectric constant. An examination of the temperature variation of the two quantities leads to this conclusion. It is further confirmed as regards field variation by the fact that the relation of activity to applied field is like $\partial D / \partial E$ vs. $E$ where $\partial D / \partial E$ is merely the instantaneous value of the dielectric constant $\kappa$. As an approximation we can write the piezo-electric modulus $\delta$ proportional to $\kappa$ :

$$
\delta=A \cdot \kappa .
$$


If this equation were exact $A$ would be a fundamental piezo-electric constant of the substance, being of the order of $1 \times 10^{-7}$ between $-20^{\circ}$ C. and $+20^{\circ}$ C. At some temperatures and for some exceptional specimens the relation does not seem to be so simple.

\section{Effect of Moisture on Piezo-electric Properties.}

In order to investigate the effect of dryness on the activity of Rochelle salt, some phosphorus pentoxide was enclosed in the chamber containing the crystal. The crystal soon started to dehydrate and after a few days was covered by a white coating. The piezo-electric throw for a load of 250 grams continually diminished. When the response was tested at different fields a more interesting fact was observed. Besides the decrease in response, the maxima were displaced along the field axis into a condition of greater polarization. This is shown by Fig. 3, the curves

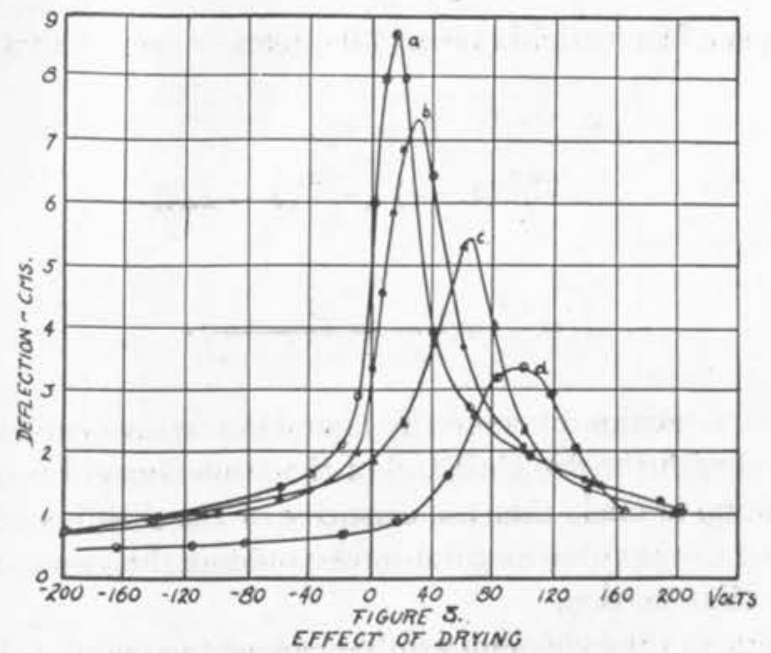

Fig. 3.

being taken after the lapse of the following times: (b) I day, (c) 3 days, (d) 12 days.

The decrease in the maxima and also their displacement is in the same direction as, and may be entirely due to, the effect of different dielectric properties of the crystal and of the dehydrated layer. In other words the presence of a layer of inactive dielectric of relatively low specific inductive capacity will diminish the charge on the plates due to the polarization of the central active layer, and thus decrease the piezoelectric response. It will also diminish the effective field across the active layer making it necessary to increase the potential difference 
between the plates to produce the same field across the inner layer, thus shifting the position of maximum activity. The effects due to uniform layers can be readily calculated. Let $P_{0}$ be the polarization produced in the middle layer by pressure, let $P_{1}$ and $P_{2}$ be the electrically induced polarizations in the dielectrics $I$ and 2 respectively (Fig. 4). Since the dielectric displacement is solenoidal we have:

$$
D^{\prime}=E_{1}+4 \pi P_{1}+4 \pi P_{0}=E_{2}+4 \pi P_{2}=4 \pi \sigma .
$$

Since

$$
P_{1}=\left(\kappa_{1}-\mathrm{I}\right) E_{1} \text { and } P_{2}=\left(\kappa_{2}-\mathrm{I}\right) E_{2}
$$

we can write

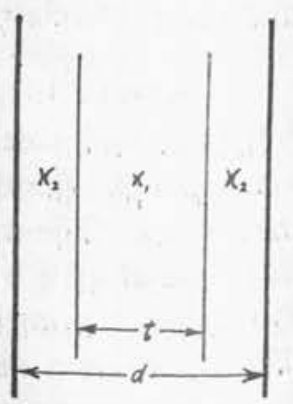

Fig. 4.

$$
D^{\prime}=\kappa_{1} E_{1}+4 \pi P_{0}=\kappa_{2} E_{2} .
$$

The difference of potential between the plates is zero so that, replacing $P_{0}$ by $\sigma_{0}$ :

giving:

$$
\begin{aligned}
0 & =E_{2}(d-t)+E_{1} t \\
& =\frac{4 \pi \sigma}{\kappa_{2}}(d-t)+\frac{4 \pi}{\kappa_{1}}\left(\sigma-\sigma_{0}\right) t,
\end{aligned}
$$

$$
\frac{\sigma}{\sigma_{0}}=\frac{Q^{\prime}}{Q}=\frac{\kappa_{2} t}{t\left(\kappa_{2}-\kappa_{1}\right)+\kappa_{1} d} .
$$

This gives us a relation between the piezo-electric response at zero field of the crystal with the dry shell and of the same crystal before it dried. The assumption is made that the elasticity of the shell is equal to that of the crystal so that a given total force produces the same polarization in the crystalline portion.

The position of the maximum will be changed to another value of total potential difference on the crystal. Let $V$ be the total potential drop and $V^{\prime}$ be the drop across the crystalline part. When there is no dehydrated layer present

$$
V=V^{\prime}=d E,
$$

where $E$ is the field strength in the dielectric. When there is a layer of uniform thickness $(d-t) / 2$ on both faces then

$$
V=(d-t) E^{\prime \prime}+t E^{\prime},
$$

where $E^{\prime \prime}$ and $E^{\prime}$ are the field strengths in the dielectrics 2 and I respectively. The dielectric displacement

$$
D=\kappa_{2} E^{\prime \prime}=\kappa_{1} E^{\prime}+4 \pi P_{0}
$$


is solenoidal, and we can eliminate $E^{\prime \prime}$ from equations above and write:

$$
V^{\prime}=d E=(d-t) \frac{\kappa_{1}}{\kappa_{2}} E^{\prime}+t E^{\prime}+4 \pi P_{0}\left(\frac{d-t}{\kappa_{2}}\right) .
$$

Since the last term is small compared to the rest of the expression, this gives:

$$
\frac{E^{\prime}}{E}=\frac{\kappa_{2} d}{t\left(\kappa_{2}-\kappa_{1}\right)+d \kappa_{1}} .
$$

The following quantities were measured and substituted in these equations.

$$
\begin{aligned}
\kappa_{1} & =1000, \quad d=0.22 \mathrm{~cm} ., \\
\kappa_{2} & =180, \quad t=0.14 .
\end{aligned}
$$

The quantity $t$ is an average obtained by breaking the crystal in several places and it is probably not very accurate because of the irregularity of the outer layer. We should, however, get a rough check on the plausibility of the proposed explanation. We find that

and that

$$
\frac{Q^{\prime}}{Q}=0.24
$$

$$
\frac{E^{\prime}}{E}=0.38
$$

While the values of $Q^{\prime} / Q$ and $E^{\prime} / E$ from the maxima of curves $a$ and $d$ of Fig. 3 are respectively 0.39 and 0.33 . The agreement is not as good as could be desired even after making allowance for the difficulties in . estimating $t$. Possibly there is a true humidity effect with respect to piezo-electric activity but the above shows, at least, that it is quite small.

\section{Piezo-electric Activity .and Temperature.}

In order to investigate the variation of activity with temperature, the chamber holding the crystal was immersed in $\mathrm{CO}_{2}$ snow. After everything was thoroughly cooled and at $-75^{\circ} \mathrm{C}$., the chamber was allowed to heat up. Above $-35^{\circ} \mathrm{C}$. an electric heater was used. It was wound on a glass jar and insulated from the crystal chamber by a felt jacket. This jar was immersed in an oil bath to steady the heating rate while the felt eliminated any rapid changes of heating of the crystal. The current was gradually increased so as to keep the rate of heating uniformly between $\frac{1}{2}{ }^{\circ}$ and $\mathrm{I}^{\circ} \mathrm{C}$. per minute so as to eliminate thermoelastic stresses. The temperature was measured by means of a copper-constantan thermocouple directly soldered to an electrode on the crystal. In this way the actual temperature of the crystal itself was measured. 
When the piezo-electric response or galvanometer throw for 250 grams was measured at the various temperatures for the first specimen the curve of Fig. 5 was obtained. This was duplicated to check the second

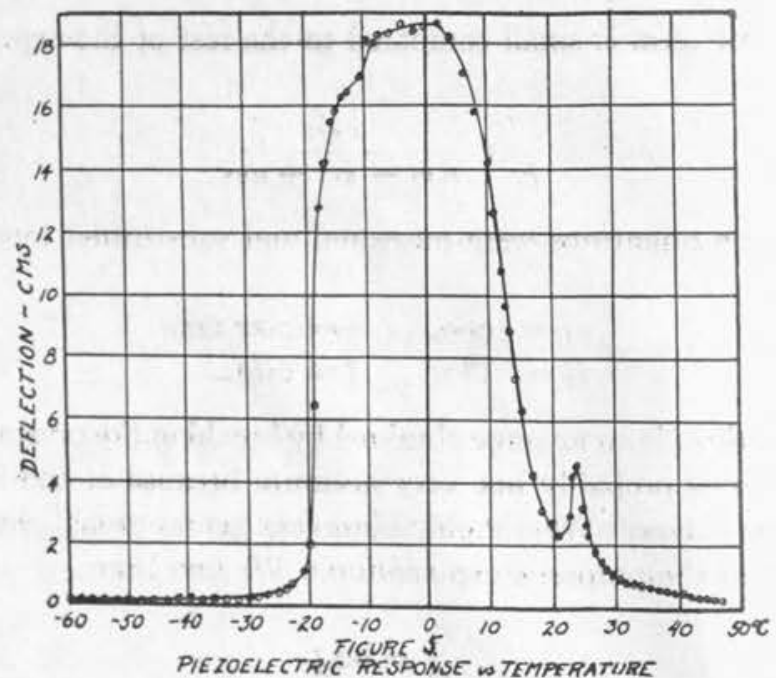

Fig. 5 .

maximum. At $-70^{\circ} \mathrm{C}$. the piezo-electric activity is comparatively negligible. As the temperature is raised slowly the activity stays small until $-30^{\circ} \mathrm{C}$. is reached. At $-20^{\circ} \mathrm{C}$. it is rising very rapidly, reaching a maximum at about $0^{\circ} \mathrm{C}$. It decreases again but at $23^{\circ} \mathrm{C}$. comes to a small but sharp maximum from which it diminishes slowly, becoming very small at $+50^{\circ} \mathrm{C}$. The magnitude of the second maximum varies with the temperature at which heating begins. This second maximum was found in the case of two crystal plates provided with tinfoil electrodes attached by shellac.

Three other crystals were prepared with electrodes of mercury held against the crystal by two rectangular cups attached by wax. The thermocouple wires were immersed in the mercury. None of the crystals so prepared gave the second maximum. Moreover, none of them were as active as those used above. The variation of piezo-electric response of these specimens is shown in Fig. 6. The increase at $-30^{\circ}$ to $-15^{\circ} \mathrm{C}$. and the decrease at $+20^{\circ} \mathrm{C}$. to $+30^{\circ} \mathrm{C}$. are remarkably consistent. Between $-15^{\circ}$ and $+20^{\circ} \mathrm{C}$, however, they each show different characteristics. These mercury electrode crystals seemed to give more constant results than the crystals with tinfoil electrodes attached by shellac.

It was then suspected that the increased response of the crystal with 


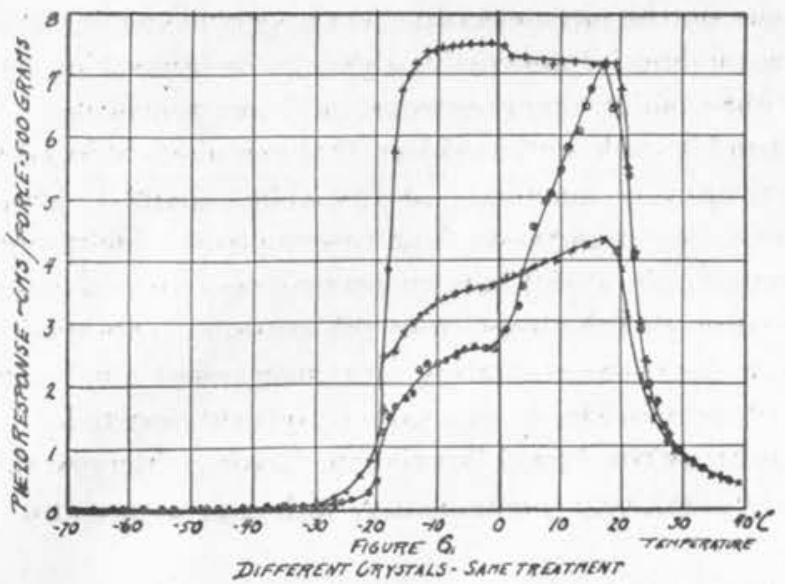

Fig. 6.

tinfoil electrodes and the presence of the second maximum was in some way due to the penetration into the crystal of the alcohol solvent of shellac. Accordingly one of the crystals originally with mercury electrodes was provided with the other type. As soon as the shellac was sufficiently dry, Curve $b$, Fig. 7 was obtained, the response originally

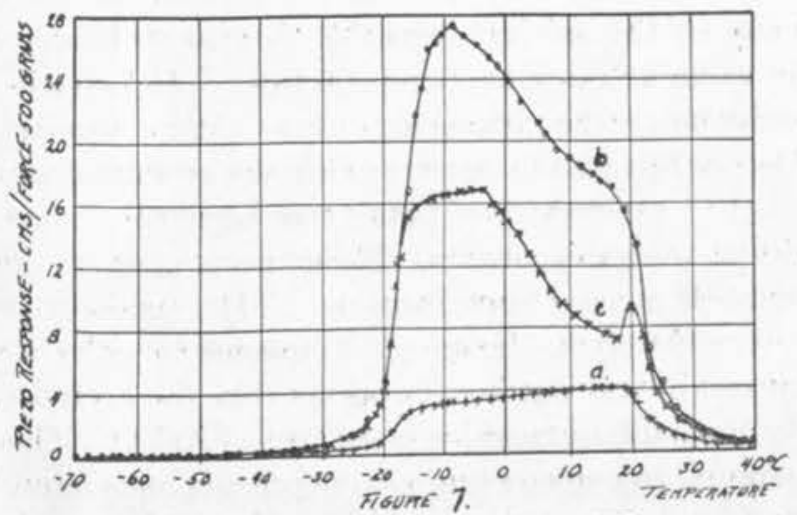

MATE CRYSTAL-DIFFERENT TREATMENT

Fig. 7.

having followed Curve $a$. The sensitivity increased seven-fold at some temperatures but there was no second maximum. In two weeks the characteristics had changed to those shown in Curve $c$, seemingly checking the suspicion that alcohol was responsible for the second maximum and for the increased sensitivity. It would be interesting to use alcohol cup electrodes and investigate the continuous effect of alcohol soaking into the crystal. T.ie effect is probably not chemical. 
In a paper on the piezo-electric effect on Rochelle salt, A. M. Nicolson ${ }^{1}$ describes a method for desiccating the crystals by soaking in alcohol and heating, thus making them stronger and more sensitive. The writer is certain, from his work on the subject, that complete desiccation will make the crystal entirely inactive. In the above method, apparently, the treatment is not prolonged enough to completely dehydrate more than a shell around the crystal and its effectiveness may be connected with the penetration of the alcohol into the crystal. The heating at $40^{\circ} \mathrm{C}$. may also be effective in allowing a rearrangement and recrystallization of some of the molecules or groups not properly oriented. W. G. Cady. as well as the writer, has observed that heating the crystal will usually increase its sensitivity permanently, although sometimes the reverse is true.

An interesting side-light on the temperature variation of piezo-electric activity is offered by a study of curves like that of Fig. 2 but at different temperatures. They show that the effect of temperature is not so much to change the piezo-electric activity as to shift the position of the maximum from one value of the field to another. This is probably connected with the variation of the dielectric constant with temperature which will be taken up presently.

The charging throws of the crystal used as a condenser show variations similar to those of the activity except that they do not tend to zero but to a constant value at the lower temperatures. The crystals giving the second maximum on the piezo-electric curve show a similar peculiarity here. The crystals with the mercury electrodes give more regular curves. At $20^{\circ}$ to $25^{\circ} \mathrm{C}$. the crystals of both types begin to conduct, causing a steady drift of the galvanometer. Experiments seem to indicate that Ohm's law holds at least approximately. The conduction was at first thought to be electrolytic because of the manner in which Rochelle salt melts. Instead of real melting it appears that the crystal dissolves in its water of crystallization which is set free at $55^{\circ} \mathrm{C}$. The desiccated crystal, however; decomposes into a tarry product and emits heavy white fumes above $150^{\circ} \mathrm{C}$., without melting. The dehydrated crystal also begins to conduct above $20^{\circ} \mathrm{C}$., making it probable that electronic and not electrolytic conduction is observed.

Measurements of conductivity were made on the natural crystal at various temperatures up to its liquefying point. The values obtained after the conductivity was sufficiently large to use a Wheatstone bridge are as follows:

${ }^{1}$ A. M. Nicolson, Proc. Am. Inst. Ele. Eng., Vol. 38, p. 13r5 (19r9). 
TABLE I.

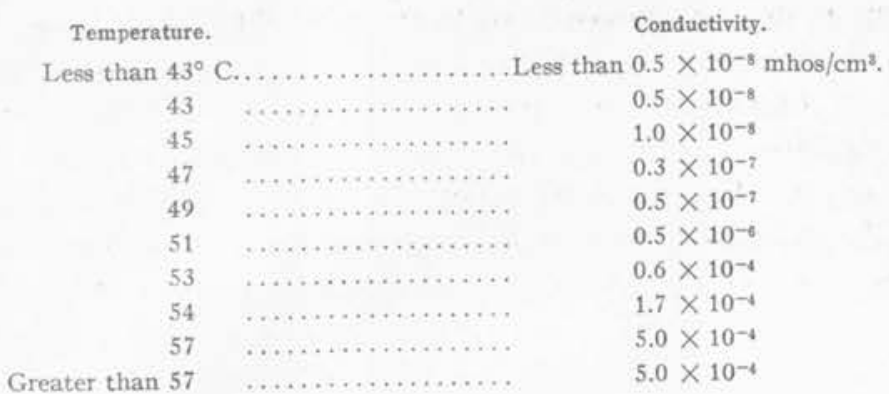

At temperatures below $20^{\circ}$ the dry crystal is a fairly good insulator having a specific conductivity of $5 \times 10^{-12} \mathrm{mhos} / \mathrm{cm}^{3}$ at $0^{\circ} \mathrm{C}$. The conductivity decreases slightly at still lower temperatures. In all these measurements the surfaces were thoroughly dried by the presence of phosphorus pentoxide in the crystal chamber.

TABLE II.

\begin{tabular}{|c|c|c|c|c|c|c|}
\hline \multirow{2}{*}{$\begin{array}{l}\text { Temp. } \\
\text { Cent. }\end{array}$} & \multicolumn{2}{|c|}{ Dielectric Constant. } & \multicolumn{4}{|c|}{ Piezoelectric Modulus. } \\
\hline & $A$ & $B$ & $A$ & & & B \\
\hline 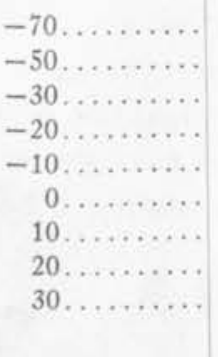 & $\begin{array}{r}71 \\
85 \\
140 \\
386 \\
943 \\
1,380 \\
1,100 \\
688 \\
423 \\
\text { Condu }\end{array}$ & $\begin{array}{r}42 \\
50 \\
146 \\
252 \\
924 \\
956 \\
928 \\
645 \\
146 \\
\text { ences }\end{array}$ & $\begin{array}{c}0.041 \\
0.068 \\
0.41 \\
5.4 \\
18.9 \\
22.9 \\
18.9 \\
13.5 \\
2.2 \\
0.41\end{array}$ & $\begin{array}{c}\times 10^{-5} \\
u \\
4 \\
4 \\
u \\
4 \\
u \\
u \\
4 \\
u\end{array}$ & $\begin{array}{l}0.017 \\
0.017 \\
0.065 \\
1.08 \\
6.07 \\
6.75 \\
7.42 \\
8.10 \\
1.08 \\
0.41\end{array}$ & $\begin{array}{c}\times 10^{-5} \\
" \\
u \\
" \\
" 6 \\
" \\
" \\
" 4 \\
"\end{array}$ \\
\hline
\end{tabular}

Table II. gives values of the dielectric constants for a field changing from o to 880 volts $/ \mathrm{cm}$, and of the piezo-electric modulus $\delta_{14}$ for shearing stresses of $220 \mathrm{grams} / \mathrm{cm}^{2}{ }^{2}$. The modulus $\delta_{14}$ is defined by the relation given by Voigt $\sigma_{1}=-\delta_{14} Y_{z}$ where $\sigma_{1}$ is the surface density of charge and $Y_{z}$ is the shearing stress producing it. The given values are thought to be the most representative in each case. They are subdivided into two classes, according to whether the electrodes were tinfoil attached by shellac (column $A$ ) or mercury in direct contact with the crystal (column B). The former method is the most convenient to use in general practice, but the latter is thought to give more exactly the properties of Rochelle salt in the direction of the $a$ axis. The dielectric 
constants are surprisingly large, a fact noticed by Pockels ${ }^{1}$ who supposes that this is due to "internal conductivity." The writer however has measured separately the conductivity at these temperatures and is of the opinion that this is a true dielectric constant arising from polarization of the dielectric, and for this reason being so closely related to the piezoelectric effect. Because of the relatively low specific inductive capacity of the desiccated crystal it is thought that the high specific inductive capacity of Rochelle salt is partly due to the water molecule.

\section{Magnetic Analogy.}

There seems to be a strong analogy between the behavior of Rochelle salt as a dielectric possessing hysteresis and having an exceptionally large dielectric constant, and the phenomena of ferromagnetism. Accordingly some of the features of Weiss's theory of magnetism may find their counterpart in the phenomena in Rochelle salt. Weiss ${ }^{2}$ plots the susceptibility against the reciprocal of the absolute temperature and finds that the curve may be represented by a succession of straight lines. He interprets the sudden changes in slope as due to changes in the number of magnetons. If the data of Figs. $8 a$ and 12 are plotted against the reciprocal of the absolute temperature one likewise gets what may be considered to be a succession of straight lines. Actually however there occur rounded corners where the curves suddenly change direction. This may be due to slight non-uniformity in heating which occurred in spite of the precautions taken. It is considered that the straight portions are at least as definite as those shown in Weiss's paper. The most abrupt changes are at $-20^{\circ} \mathrm{C}$. and at $+20^{\circ} \mathrm{C}$. These may accordingly be considered as the "Curie points" in Rochelle salt.

\section{Relation to Optical Propertifs.}

Some of the optical properties of Rochelle salt can be at least approximately found from the electrical data given. In the course of this calculation it is of course necessary to introduce some assumptions notably as to the nature of the permanent polarization. If one knew just how the permanent polarization was pioduced he could find the free period of this mechanism. The data needed are the force per unit displacement $f$ and the mass $m$ of that part of the molecule. The wave-length corresponding to the free period is given by the expression

$c$ being the velocity of light.

$$
\lambda=2 \pi c \sqrt{\frac{m}{f}}
$$

\footnotetext{
${ }^{1}$ Pockels, Lehrbuch der Krystaloptik, p. 508.

2P. Weiss. J. de Physique, Vol. I, p. 968 (rgr 1 ).
} 
Let us assume for simplicity that only one electron is involved in the creation of the permanent moment. The quantity $f$ can be derived from the value of the permanent moment $P_{0}=30$ e.s.u. $/ \mathrm{cm}^{2}$ and from the displacement of the electron producing it. Since the force on an electron inside a dielectric of polarization $P_{0}$ is roughly equal to $\frac{1}{3} P_{0} e^{1}$ the expression for the wave-length may be put in the form:

$$
\lambda=2 \pi c \sqrt{\frac{3^{m d}}{P_{0} e}} .
$$

The value of $d$, the displacement of the electron, can be found as follows:

Taking 30 e.s.u. $/ \mathrm{cm}^{2}$ as the natural polarization, the moment per molecule is obtained by dividing by the number of molecules per c.c., the result being $7 . \mathrm{I} \times 10^{-21}$ e.s.u. In each of these molecules there are 140 electrons, this being the sum of the atomic numbers of the constituent elements in Rochelle salt. If we suppose as above that only one of these is effective in producing the piezo-electric moment, its displacement from the center of force of the rest of the molecule will be $d=2.7 \times \mathrm{rO}^{-11} \mathrm{~cm}$. It would of course be more reasonable to suppose that at least several of the electrons are displaced by different amounts, and to the extent that we do this the value calculated above becomes smaller.

Using these values of $P_{0}$ and $d$ for the simple case treated above we find for the wave-length the value:

$$
\lambda=4.2 \mu \text {. }
$$

Coblent $\mathrm{z}^{2}$ shows the presence of fairly strong absorption in this region of the infra-red. This may, however, be due to the water of crystallization and not to the cause cited above. These two possibilities should be distinguishable experimentally because the character of the absorption due to these electrons should change greatly with the temperature, as the piezo-electric elasticity or force per unit displacement of the electrons changes.

The natural period as found above should be the same as that involved in rotatory dispersion formulæ, since both the piezo-electric effect and optical rotation are due to an unsymmetrical or twisted structure of the molecule. J. J. Thomson ${ }^{3}$ gives an approximate formula for the specific rotation, namely:

$$
\rho=\frac{e^{2} d p^{2}}{c^{2} M m n^{2}},
$$

${ }^{1}$ H. A. Lorentz, Theory of Electrons, p. 306.

:W. W. Coblentz, Infra Red Spectra, Vol. 2, p. 38.

a J. J. Thomson, Phil. Mag., Dec., I920. 
in which $e$ is the charge of the electron, $c$ is the velocity of light and $M$ and $m$ are the masses of the molecule and of the electron, $d$ is the radius of the molecule, $p$ is the free period, and $n$ is the frequency for which $\rho$ is to be calculated. Using the value of $p$ derived from piezo-electric data we find for sodium light the specific rotation of the order of magnitude of $10^{\circ}$, the tables giving $22.1^{\circ}$. Considering the fact that so little is known of the structure of the Rochelle salt molecule, the approximation is fair.

The writer is indebted to Professor W. F. G. Swann, who initiated this research and gave many helpful suggestions, and to Dr. W. R. Whitney, Director of the Research Laboratory of the General Electric Company, whose presentation of two beautiful crystals made the work possible.

Physical Laboratory,

UNIVERSITY OF MINNESOTA. 